\title{
Electrical transport of an AlGaN/GaN two-dimensional electron gas
}

\author{
A. Saxler, ${ }^{\text {a) }}$ P. Debray, ${ }^{\text {a,b) }}$ R. Perrin,${ }^{\text {a) }}$ S. Elhamri, ${ }^{\text {a,c) }}$ W. C. Mitchel,${ }^{\text {a) }}$ C.R. Elsass, ${ }^{\text {d) }}$ \\ I.P. Smorchkova, ${ }^{\text {() }}$ B. Heying, ${ }^{\text {() }}$ E. Haus, ${ }^{\text {d) }}$ P. Fini, ${ }^{\text {d) }}$ J.P. Ibbetson, ${ }^{\text {() }}$ S. Keller, ${ }^{\text {() }}$ P.M. \\ Petroff, ${ }^{\text {d) }}$ S.P. DenBaars, ${ }^{\text {d) }}$ U.K. Mishra, ${ }^{\text {d) }}$ and J.S. Speck ${ }^{\text {d) }}$ \\ ${ }^{a}$ Air Force Research Laboratory, Materials and Manufacturing Directorate, \\ AFRL/MLPO, Wright-Patterson AFB, Ohio 45433-7707

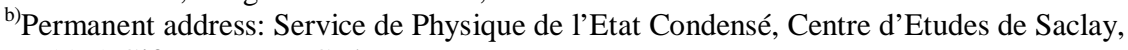 \\ F-91191 Gif-sur-Yvette Cedex, France. \\ ${ }^{c}$ Permanent address: Department of Physics, University of Dayton, Dayton, OH 45469. \\ ${ }^{d)}$ College of Engineering, University of California, Santa Barbara, CA 93106
}

\begin{abstract}
An $\mathrm{Al}_{\mathrm{x}} \mathrm{Ga}_{1-\mathrm{x}} \mathrm{N} / \mathrm{GaN}$ two-dimensional electron gas structure with $\mathrm{x}=0.13$ deposited by molecular beam epitaxy on a GaN layer grown by organometallic vapor phase epitaxy on a sapphire substrate was characterized. Hall effect measurements gave a sheet electron concentration of $5.1 \times 10^{12} \mathrm{~cm}^{-2}$ and a mobility of $1.9 \times 10^{4} \mathrm{~cm}^{2} / \mathrm{Vs}$ at 10 K. Mobility spectrum analysis showed single-carrier transport and negligible parallel conduction at low temperatures. The sheet carrier concentrations determined from Shubnikov-de Haas magnetoresistance oscillations were in good agreement with the Hall data. The electron effective mass was determined to be $0.215 \pm 0.006 \mathrm{~m}_{0}$ based on the temperature dependence of the amplitude of Shubnikov-de Haas oscillations. The quantum lifetime was about one-fifth of the transport lifetime of $2.3 \times 10^{-12} \mathrm{~s}$.
\end{abstract}

\section{INTRODUCTION}

Many applications exist for the III-Nitrides in high-power and high-temperature electronics, ${ }^{1-3}$ solar-blind ultraviolet photodetectors, ${ }^{4}$ and blue and ultraviolet light emitting and laser diodes. ${ }^{5}$ A structure of particular interest for the electronic devices is the $\mathrm{AlGaN} / \mathrm{GaN}$ two-dimensional electron gas (2DEG) for use in high electron mobility transistors (HEMTs). In this paper, we study in detail the electrical transport properties of a high-mobility AlGaN/GaN 2DEG. Temperature and magnetic field dependent Hall effect measurements are used to study the basic transport properties. Temperature dependent Shubnikov - de Haas $(\mathrm{SdH})$ measurements permitted the extraction of the electron effective mass in GaN. Determination of this constant is important for use in device modeling, but there is considerable scatter in the reported data for the electron effective mass which ranges from $0.18-0.23 \mathrm{~m}_{0}{ }^{6-9}$ Two previous reports of the effective mass using $\mathrm{SdH}$ measurements for AlGaN/GaN 2DEGs have been made. ${ }^{6,7}$ The $\mathrm{SdH}$ measurement for a structure grown on a SiC substrate ${ }^{6}$ yielded a significantly different result than that measured by other techniques. In this paper, we will use the $\mathrm{SdH}$ measurements to estimate the electron effective mass, scattering time, and carrier concentration and compare the results to those obtained by the Hall effect measurements.

\section{EXPERIMENT}

An $\mathrm{Al}_{\mathrm{x}} \mathrm{Ga}_{1-\mathrm{x}} \mathrm{N} / \mathrm{GaN}$ two-dimensional electron gas structure was deposited by molecular beam epitaxy on a 2-3 $\mu \mathrm{m}$ thick GaN layer grown by organometallic vapor

F99W11.10 
phase epitaxy on a sapphire substrate. The $\mathrm{Al}_{\mathrm{x}} \mathrm{Ga}_{1-\mathrm{x}} \mathrm{N}$ was approximately $50 \mathrm{~nm}$ thick. The details of the growth have been reported previously. ${ }^{10}$

Hall effect measurements were performed over a temperature range of $10-300$ $\mathrm{K}$ and a magnetic field range of $0-2 \mathrm{~T}$. Both the Hall effect and the SdH measurements were taken in the four-probe van der Pauw configuration using annealed Ti/Al contacts placed at the four corners of a square.

Measurements of $\mathrm{SdH}$ magnetoresistance oscillations were performed using a low ac bias current to avoid electron heating. A magnetic field of $0-9 \mathrm{~T}$ was used. The temperature was controlled from $1.2-4.2 \mathrm{~K}$ by immersing the sample in liquid helium and setting the pressure. A calibrated thermometer was then used to measure the temperature.

\section{RESULTS AND DISCUSSION}

As seen in Fig. 1, the sheet electron concentration and mobility measured by the Hall effect are nearly independent of temperature below about $100 \mathrm{~K}$. This behavior is typical of two dimensional electron gas structures. At $10 \mathrm{~K}$, the electron concentration, $n_{H}$, was $5.06 \times 10^{12} \mathrm{~cm}^{-2}$, and the Hall mobility, $\mu_{H}$, was $1.91 \times 10^{4} \mathrm{~cm}^{2} / \mathrm{Vs}$. The transport scattering time, $\tau_{c}=\mu_{H} m^{*} / e$, where $m^{*}$ is the effective mass and $e$ is the electron charge, was found to be 2.34 ps.

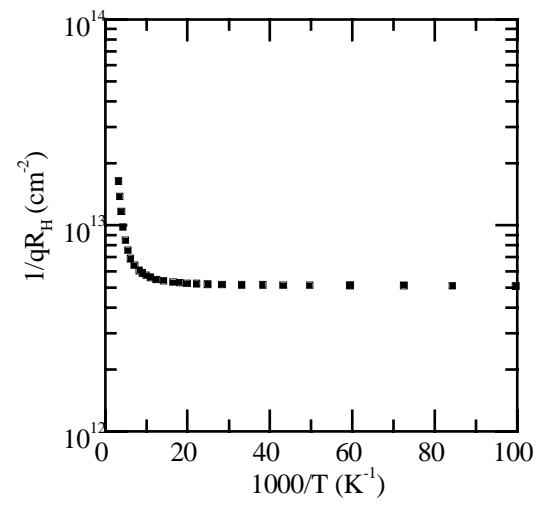

(a) temperature. (b) Hall mobility $\mu_{H}$ as a function of temperature.

In order to determine if there was any significant parallel conduction at low temperature, magnetic field dependent Hall effect measurements were taken. The data taken at $10 \mathrm{~K}$ is plotted in Fig. 2 (a) along with the fit as in the analysis of Kim et al. ${ }^{11}$ The reduced conductivities, $\mathrm{X}$ and $\mathrm{Y}$, are $\sigma_{\mathrm{xx}}$ and $2 \sigma_{\mathrm{xy}}$ respectively divided by the zero field conductivity. When the reduced conductivities are plotted in this manner and only a single electron mobility $\mu$ is present, at a magnetic field $\mathrm{B}=1 / \mu, \mathrm{X}$ passes through 0.5 and $\mathrm{Y}$ peaks with a magnitude of 1 . It is clear from this data that there is only one singlecarrier conduction path at $10 \mathrm{~K}$. The single carrier fit to this data gives an electron 
concentration of $5.08 \times 10^{12} \mathrm{~cm}^{-2}$ and a mobility of $1.90 \times 10^{4} \mathrm{~cm}^{2} / \mathrm{Vs}$, in very good agreement with the single magnetic field Hall taken at $0.5 \mathrm{~T}$. The mobility spectrum shown in Fig. 2(b) was obtained using the magnetic field dependent data of Fig. 2(a) processed with software from Lake Shore Cryotronics using the quantitative mobility spectrum analysis technique. ${ }^{12}$ This plot also illustrates that there is a single dominant high mobility channel. Fig. 2(c) shows the reduced conductivity at a higher temperature of $160 \mathrm{~K}$. From this plot it is apparent that there is more than one conduction path in the sample since $Y$ does not reach -1 . A two-carrier fit gives $n_{1}=5.0 \times 10^{12} \mathrm{~cm}^{-2}, \mu_{1}=0.82 \mathrm{x}$ $10^{4} \mathrm{~cm}^{2} / \mathrm{Vs}, \mathrm{n}_{2} \sim 3 \times 10^{13} \mathrm{~cm}^{-2}$, and $\mu_{2} \sim 2 \times 10^{2} \mathrm{~cm}^{2} / \mathrm{Vs}$ and is plotted as a line. The mobility spectrum also shows a high mobility channel and a lower mobility channel with significantly lower conductivity. The limited magnetic field range is responsible for reduced certainty in determining the lower mobility. The second lower mobility channel can be interpreted as the bulk-like unintentionally-doped $\mathrm{GaN}$ layer which freezes out at low temperatures.

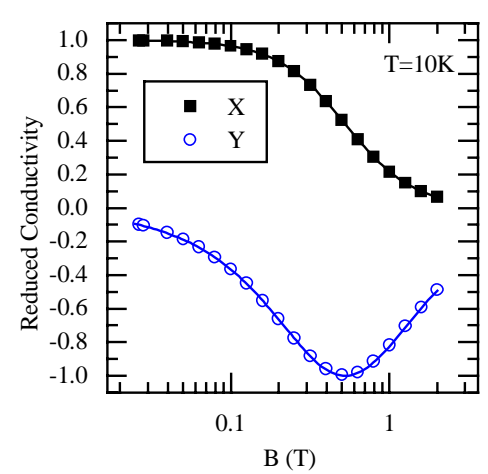

(a)

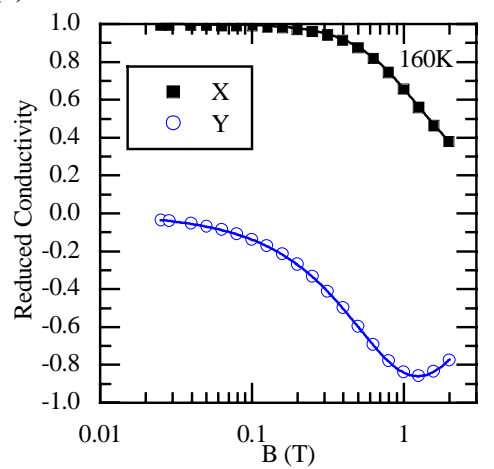

(c)

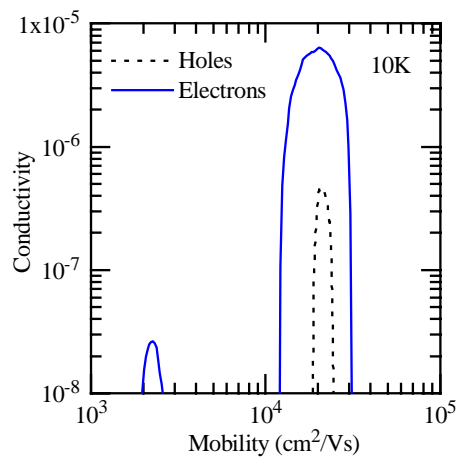

(b)

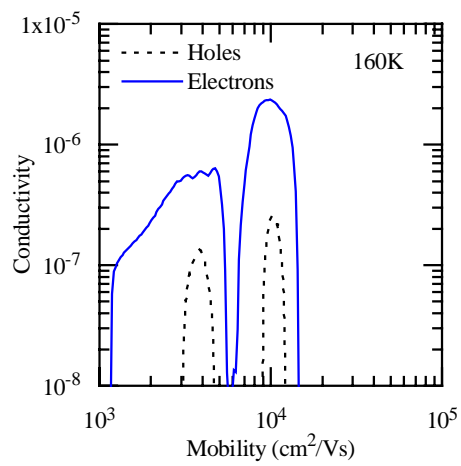

(d)

Figure 2. Reduced conductivity as a function of magnetic field (a), and mobility spectrum (b) at $10 \mathrm{~K}$ showing that a single-carrier high mobility channel dominates the conduction; and $160 \mathrm{~K}$ (c),(d) showing the presence of a second lower mobility channel . 
The $\mathrm{SdH}$ oscillations in the magnetoresistance $\mathrm{R}_{\mathrm{xx}}(\mathrm{B})$ were measured at $1.3 \mathrm{~K}$ along two mutually perpendicular sides (labeled R1 and R2) of the square van der Pauw sample. A "beating" effect was observed in the amplitude of the R1, as would be expected from two interfering oscillations of slightly different frequencies. Assuming only one subband is occupied and neglecting the contribution of higher harmonics, the oscillatory part $\Delta \rho_{\mathrm{xx}}$ of the magnetoresistivity can be expressed as: ${ }^{13}$

$$
\frac{\Delta \rho_{x x}}{2 \rho_{0}}=2 \frac{\chi}{\sinh (\chi)} \exp \left(\frac{-\pi}{\omega_{c} \tau_{q}}\right) \cos \left(\frac{2 \pi \varepsilon}{\hbar \omega_{c}}-\pi\right)
$$

where $\chi=2 \pi^{2} k_{B} T / \hbar \omega_{c}, \omega_{c}=e B / m^{*}, \varepsilon=\pi \hbar^{2} n / m^{*}$ is the Fermi energy, $\tau_{\mathrm{q}}$ is the quantum scattering time, $B$ is the magnetic field, $k_{B}$ is the Boltzmann constant, $T$ is the absolute temperature, $k_{B}$ is the reduced Plank constant, and $n$ is the sheet electron concentration of the 2DEG. From this equation and the observed magnetic field and temperature dependent magnetoresistance data we will extract the carrier concentration, the effective mass, and the quantum scattering time. This is possible since the magnetoresistance is directly proportional to the magnetoresistivity through a sample geometrical factor.

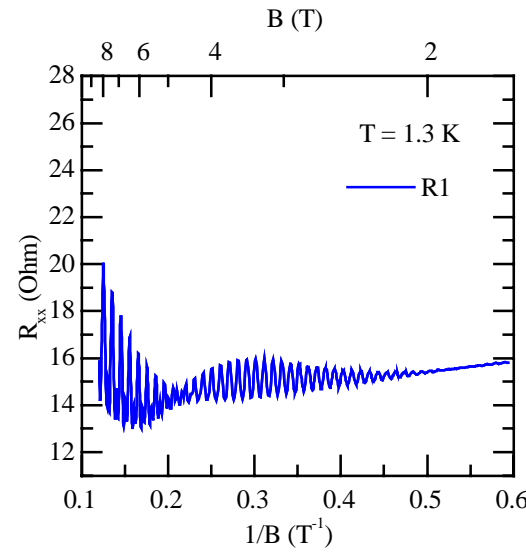

(a)

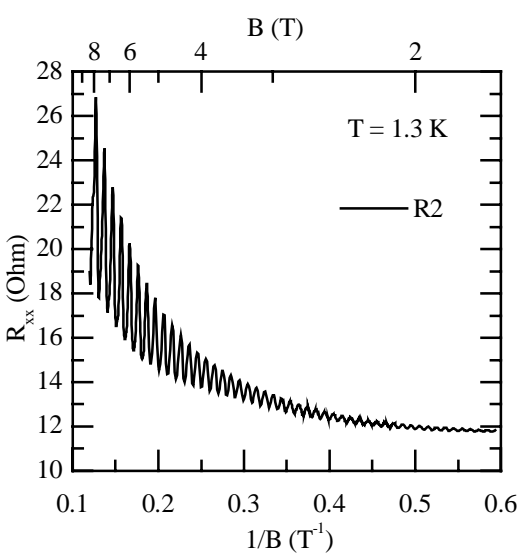

(b)

Figure 3. Shubnikov-de Haas oscillations at $1.3 \mathrm{~K}$ from two mutually perpendicular sides (a) $R 1$ and (b) $R 2$ of the van der Pauw sample. Notice the attenuation of $\mathrm{R} 1$ amplitudes due to a "beating" effect.

Since the observed SdH oscillations do not show the presence of higher harmonics, Eq. 6 can be used to obtain the carrier concentration from the frequency of the oscillations in 1/B. Fig. 4(a) shows the inverse magnetic field at which the peak maxima occur. The sheet carrier concentrations for the R1 and R2 oscillations were found to be 4.77 and $4.94 \times 10^{12} \mathrm{~cm}^{-2}$, respectively. The corresponding drift mobilities determined using the observed value of $\rho_{\mathrm{o}}$ were 2.03 and $1.96 \times 10^{4} \mathrm{~cm}^{2} / \mathrm{Vs}$. Interestingly, the frequency difference determined from the beating period of the R1 oscillations is found 
to correspond to an electron concentration difference of about $0.18 \times 10^{12} \mathrm{~cm}^{-2}$. The sheet carrier concentrations obtained from the $\mathrm{SdH}$ oscillations are slightly lower than that of the Hall value which may indicate the presence of a very small parallel conduction. The background rise of the R2 oscillations (Fig. 8) indicates field-induced localization of some donor impurities, consistent with small parallel conduction. The small anisotropies observed in the carrier concentration and mobility may result from a slight inhomogeneity in the sample, especially because in the van der Pauw configuration the current paths are not necessarily linear. The observation of the beating effect may also result from this inhomogeneity.

The effective mass can be extracted by measuring the amplitude of the $\mathrm{SdH}$ oscillations as a function of temperature. In Fig. 4(b), the natural logarithm of the amplitude $A$ divided by temperature $T$ is plotted as a function of the temperature. The data was fit simultaneously for two different resistance configurations (labeled R1 and $\mathrm{R} 2)$ and two different magnetic fields ( $3 \mathrm{~T}$ and $4 \mathrm{~T})$. The value obtained from the fit was $0.215 \pm 0.006 \mathrm{~m}_{0}$ which is in good agreement with recently reported values obtained from other techniques such as cyclotron resonance. ${ }^{7-9}$

Fig. 4(c) is a "Dingle plot" of the R2 oscillation amplitudes (Fig. 8). The quantum relaxation time $\tau_{\mathrm{q}}$ was obtained by fitting the data. Since the amplitudes of R1 oscillations are attenuated by the beating effect, they were not used. The rather poor fit may indicate that some beating is also present in the R2 oscillations. The fit yielded a value of about $0.5 \mathrm{ps}$ for the quantum scattering time, which is significantly lower than the transport scattering time $\tau_{\mathrm{p}}$ of 2.34 ps obtained in the previous section. Note that $\tau_{\mathrm{q}}$ is given by the total scattering rate, whereas $\tau_{\mathrm{p}}$ is weighted by the scattering angle in such a way so as to minimize low-angle scattering. For the $2 \mathrm{DEG}$ at the heterointerface, we expect the dominant scattering to be due to long-range potentials associated with donor impurities outside the well which produce predominantly small-angle scattering. A value of $\tau_{\mathrm{p}}$ higher than $\tau_{\mathrm{q}}$ is therefore expected and has often been observed. ${ }^{20}$

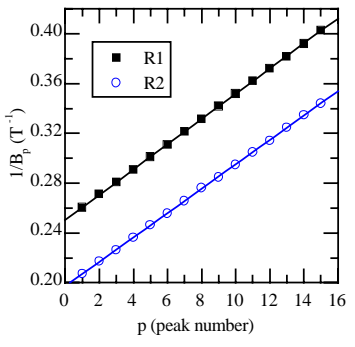

(a)

4. (a) Peak positions in reciprocal magnetic field of SdH oscillations and fit to obtain the carrier concentrations. (b) Fitting for the effective mass for two magnetic field values and two resistivity configurations simultaneously gives $\mathbf{m}^{*}=$ $0.215 \pm 0.006 \mathrm{~m}_{0}$. (c) Dingle plot for determination of scattering time by fitting the field dependent amplitude.

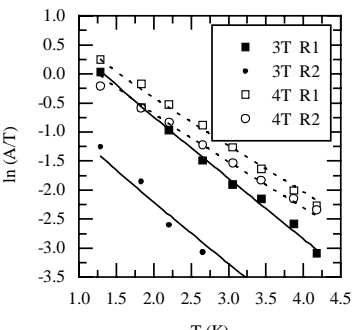

$\mathrm{T}(\mathrm{K})$

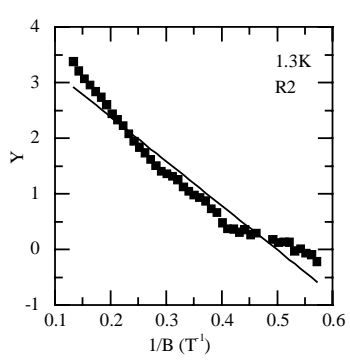

(c)$$
\text { . }
$$ 


\section{CONCLUSIONS}

An $\mathrm{Al}_{\mathrm{x}} \mathrm{Ga}_{1-\mathrm{x}} \mathrm{N} / \mathrm{GaN}$ two-dimensional electron gas structure with $\mathrm{x}=0.13$ was deposited by molecular beam epitaxy on a GaN layer grown by organometallic vapor phase epitaxy on a sapphire substrate. Mobility spectrum analysis showed negligible parallel conduction at low temperatures. The sheet carrier concentrations determined from $\mathrm{SdH}$ magnetoresistance oscillations were in good agreement with the Hall data. The uncorrected electron effective mass was determined to be $0.215 \pm 0.006$ based on the temperature dependent amplitude of the $\mathrm{SdH}$ oscillations. The quantum lifetime was found to be about one-fifth of the transport lifetime of $2.3 \times 10^{-12} \mathrm{~s}$. We have observed small anisotropies in the electronic properties of the $2 \mathrm{DEG}$ at the $\mathrm{AlGaN} / \mathrm{GaN}$ heterointerface. We currently attribute this anisotropy to a slight inhomogeneity in the sample.

\section{ACKNOWLEDGMENTS}

The work at UCSB was supported in part by the MRL Central Facilities supported by the NSF under Award No. DMR-9123048. The authors also gratefully acknowledge G. Landis, S. Davidson, A. C. Gossard, J. English, the ONR IMPACT MURI Center at UCSB (C. Wood and J. Zolper contract monitors), AFOSR (G. Witt and D. Johnstone), the Center for Quantized Electronic Structures, HRL Laboratories, the UC-MICRO program, and the NDSEG Fellowship Program.

\section{REFERENCES}

${ }^{1}$ O. Aktas, Z. F. Fan, S. N. Mohammed, A. E. Botchkarev, and H. Morkoç, Appl. Phys. Lett. 69, 3872 (1996).

${ }^{2}$ U.K. Mishra, Y.-F. Wu, B.P. Keller, S. Keller and S.P. DenBaars, IEEE Trans. on

Microwave Theory and Tech., 46, 756 (1998).

${ }^{3}$ L. McCarthy, P. Kozodoy, M. Rodwell, S. DenBaars, and U. Mishra, Compound Semiconductor 4(8), 16 (1998).

${ }^{4}$ M. Razeghi and A. Rogalski, J. Appl. Phys. 79, 7433 (1996).

${ }^{5}$ S. Nakamura, M. Seno, S. Nagahama, N. Iwasa, T. Yamada, T. Matsushita, Y. Sugimoto, and H. Kiyoku, Appl. Phys. Lett. 69, 4056 (1996).

${ }^{6}$ S. Elhamri, R. S. Newrock, D. B. Mast, M. Ahoujja, W. C. Mitchel, J. M. Redwing, M. A. Tischler, and J. S. Flynn, Phys. Rev. B 57, 1374 (1998).

${ }^{7}$ L. W. Wong, S. J. Cai, R. Li, K. Wang, H. W. Jiang, and M. Chen, Appl. Phys. Lett. 73, 1391 (1998).

${ }^{8}$ J. S. Im, A. Moritz, F. Steuber, V. Härle, F. Scolz, and A. Hangleiter, Appl. Phys. Lett. 70, 631 (1997).

${ }^{9}$ S. W. King, C. Ronning, R. F. Davis, M. C. Benjamin, and R. J. Nemanich, J. Appl. Phys. 84, 2086 (1998).

${ }^{10}$ C.R. Elsass, I.P. Smorchkova, B. Heying, E. Haus, P. Fini, K. Maranowski, J.P. Ibbetson, S. Keller, P.M. Petroff, S.P. DenBaars, U.K. Mishra, and J.S. Speck, Appl. Phys. Lett. 74, 3528 (1999).

${ }^{11}$ J. S. Kim, D. G. Seiler, and W. F. Tseng, J. Appl. Phys. 73, 8324 (1993).

${ }^{12}$ I. Vurgaftman, J. R. Meyer, C. A. Hoffman, D. Redfern, J. Antoszewski, L. Faraone, and J. R. Lindemuth, J. Appl. Phys. 84, 4966 (1998).

${ }^{13}$ P. T. Coleridge, R. Stoner, and R. Fletcher, Phys. Rev. B 39, 1120 (1989); A. Isihara and L. Smrcka, J. Phys. C 19, 6777 (1986). 